\title{
Against Euler's Proof of the Binomial Theorem for Negative and Fractional Fxponents.
}

By R. F. Muirhead, M.A., B.Sc.

In the proof of the binomial theorem for negative and fractional indices given in many text-books of algebra, and attributed to Euler, one step seems to me to involve a very gross assumption.

The symbol $f(m)$ having been taken to denote the series

$$
1+m x+\frac{m(m-1)}{1.2} x^{2}+\frac{m(m-1)(m-2)}{1.2 .3} x^{3}+\ldots \ldots
$$

it is pointed out that whenever $m$ and $n$ are positive integers we know that

$$
f(m) \times f(n) \equiv f(m+n) ;
$$

and the conclusion is drawn that since this is true for all positive integral values of $m$ and $n$, by the "permanence of equivalent forms" (whatever that may mean) we can conclude that it is true also for negative and fractional values of $m$ and $n$, whenever $f(m)$ and $f(n)$ are convergent.

If instead of the above definition of $f(m)$ we take $f(m)$ to denote

$$
\left\{1+m x+\frac{m(m-1)}{1.2^{2}} x^{2}+\ldots\right\}\{1-\phi(x) \sin m \pi\}+\psi(x) \sin m \pi,
$$

$\phi(x)$ and $\psi(x)$ being arbitrary functions, we have, when $m$ is an integer, $f(m)=(1+x)^{m}$ and by exactly the same reasoning as that referred to we can conclude that in this case also $f(m) \times f(n) \equiv f(m+n)$ for all values of $m$ and $n$, which is obviously false for most values of $\phi(x)$ and $\psi(x)$.

There must be a flaw in the reasoning: and that clearly is the assumption that an identity true for an infinite number of values of the arguments under certain restrictions as to those values, is necessarily true when these restrictions are removed. Simple examples showing the falsity of the assumption could easily be multiplied: thus $\sin (\theta+m \pi)=\cos m \pi \sin \theta$ for all integral values of $m$. But the equation is not true for fractional values of $m$. 
The retention of the bad reasoning in some of our text-books is the less to be excused, as there exists an unexceptionable proof by means of the theorem of Vandermonde, as given in C. Smith's Algebra and others.

If circumstances should render it impracticable in any case to assume a knowledge of Vandermonde's Theorem in teaching the Binomial Theorem, then one might adopt the honest course of confessing the gap in the proof, making no attempt to juggle with the "principle" of the "permanence of equivalent forms," but merely pointing out that the identity $f(m) \times f(n) \equiv f(m+n)$ can be verified to as many terms as we please.

\section{Note on Continued Fractions.}

By R. F. Moirhead, M.A., B.Sc.

1. It is usual to deduce most of the properties of Continued Fractions from the recurrence formulas

$$
\begin{aligned}
& p_{n}=a_{n} p_{n-1}+p_{n-2} \\
& q_{n}=a_{n} q_{n-1}+q_{n-2},
\end{aligned}
$$

connecting successive convergents of the fraction

$$
a_{1}+\frac{1}{a_{2}+} \quad \frac{1}{a_{3}+} \ldots \ldots .
$$

In this Note, I treat one or two theorems from a different point of view.

It is very obvious, by the formation of the Continued Fraction, that the convergents are alternately greater and smaller than those preceding and following them; the formal proof may be put as follows : - 
In the Continued Fraction

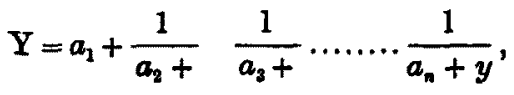

where $y$ for the present is arbitrary, writing

$$
\begin{aligned}
& Y_{n-1} \equiv a_{n}+y, \quad Y_{n-2} \equiv a_{n-1}+\frac{1}{Y_{n-1}}, \quad Y_{n-3} \equiv a_{n-2}+\frac{1}{Y_{n-2}} \ldots \ldots \ldots \ldots . . . . . . \\
& Y_{1} \equiv a_{2}+\frac{1}{Y_{2}}, \quad Y \equiv a_{1}+\frac{1}{Y_{1}} ;
\end{aligned}
$$

it is obvious that as $y$ increases $Y_{n-1}$ increases,

as $Y_{n-1}$ increases $Y_{n-2}$ decreases,

as $Y_{n \rightarrow 2}$ decreases $Y_{n \rightarrow 3}$ increases,

and so on, so that $Y$ increases with $y$ if $n$ is odd, but if $n$ is even $Y$ decreases as $y$ increases.

Now consider the fraction

$$
\mathrm{X} \equiv a_{1}+\frac{1}{a_{2}+} \quad \frac{1}{a_{3}+\ldots . .}
$$

and let $\mathrm{X} \equiv a_{1}+\frac{1}{\mathrm{X}_{1}}, \quad \mathrm{X}_{1}=a_{2}+\frac{1}{\mathrm{X}_{2}}, \quad$ etc.

(as in Chrystal's Algebra), and let $p_{1} / q_{1}, p_{2} / q_{2} \ldots$ be the convergents of the fraction $\mathrm{X}$. We see that corresponding to the values

$$
y=0, \quad y=\frac{1}{\mathrm{X}_{n}}, \quad y=\frac{1}{a_{n+1}},
$$

we have the values $\quad \mathrm{Y}=p_{n} / q_{n}, \quad \mathrm{Y}=\mathrm{X}, \quad \mathbf{Y}=p_{n+1} / q_{n+1}$.

Hence $\frac{p_{n}}{q_{n}}, \mathrm{X}$, and $\frac{p_{n+1}}{q_{n+1}}$ are in ascending or descending order of magnitude according as $\mathrm{n}$ is odd or even.

2. To show by this method that each convergent is closer to $X$ than the preceding one, we need this Lemma:

If $a, b, c$ are three positive magnitudes such that $b$ lies between $c$ and $a$, but nearer to $c$, and also $1 / b$ lies nearer to $1 / c$ than to $1 / a$, then the three magnitudes

$$
\mathrm{A} \equiv m+\frac{r}{a}, \quad \mathrm{~B} \equiv m+\frac{r}{b}, \quad \mathrm{C} \equiv m+\frac{r}{c}
$$

satisfy the same conditions among themselves, $m$ and $r$ being any positive quantities.

For obviously B lies between $\mathbf{A}$ and $\mathbf{C}$. 
Then

$$
\frac{\mathrm{B} \sim \mathrm{O}}{\mathrm{A} \sim \mathrm{B}}=\left(\frac{1}{c} \sim \frac{1}{b}\right) \div\left(\frac{1}{b} \sim \frac{1}{a}\right) .
$$

which by hypothesis is $<1$, so that $B$ is nearer to $C$ than to $A$.

Again

$$
\begin{aligned}
& \left(\frac{1}{\mathrm{C}} \sim \frac{1}{\mathrm{~B}}\right) \div\left(\frac{1}{\mathrm{~B}} \sim \frac{1}{\mathrm{~A}}\right)=\frac{\mathrm{A}(\mathrm{B} \sim \mathrm{C})}{\mathrm{C}(\mathrm{A} \sim \mathrm{B})} \\
= & \frac{m+\frac{r}{a}}{m+\frac{r}{c}} \cdot \frac{\frac{1}{c} \sim \frac{1}{b}}{\frac{1}{b} \sim \frac{1}{a}}=\frac{a m+r}{c m+r} \cdot \frac{b \sim c}{a \sim b} .
\end{aligned}
$$

The last formula shows that the ratio is $<1$ when $a \ngtr c$.

The last but one shows that the ratio is $<1$ when $a \nless c$.

Thus the Lemma is completely proved.

Now let $\mathrm{Y}^{\prime}, \mathrm{Y}^{\prime \prime}, \mathrm{Y}^{\prime \prime \prime}$ denote the values of $\mathrm{Y}$ when $y$ has the values $0, \frac{1}{X_{n}}$ and $\frac{1}{a_{n+1}}$, and similarly for $Y_{1}, Y_{2}$, etc.

Thus $\quad \mathrm{Y}_{n-1}^{\prime}=a_{n}, \quad \mathrm{Y}_{n-1}^{\prime \prime}=a_{n}+1 / \mathrm{X}_{n}, \quad \mathrm{Y}^{\prime \prime \prime}{ }_{n-1}=a_{n}+1 / a_{n+1}$.

It is easy to prove that $Y^{\prime}{ }_{n-1}, Y^{\prime \prime}{ }_{n-1}, Y^{\prime \prime \prime}{ }_{n-1}$ satisfy the same conditions as the quantities $a, b, c$ of the Iemma. Hence, as proved in the Lemma, $\mathrm{Y}_{n-2}^{\prime}, \mathrm{Y}^{\prime \prime}{ }_{n-2}, \mathrm{Y}^{\prime \prime}{ }_{n \rightarrow 2}$ also satisfy these conditions, and so on for the other $Y^{\prime}$ 's. Finally we find that $Y^{\prime}, Y^{\prime \prime}, Y^{\prime \prime \prime}$, which are respectively $p_{n} / q_{n}, \mathrm{X}$, and $p_{n+2} / q_{n+1}$, satisfy these conditions.

3. Thus we have proved directly, without the aid of the recurrence formulae, two of the most important properties of continued fractions. The investigation in the previous paragraphs is obviously applicable to the General Continued Fraction $a_{1}+\frac{b_{2}}{a_{2}+} \frac{b_{3}}{a_{3}+\ldots}$ so long as the $a$ 's and $b$ 's are positive.

The form of the investigation has implied that the continued fraction is terminating; but we can see that it will hold good for an infinite continued fraction, provided it converges to a definite limit. 